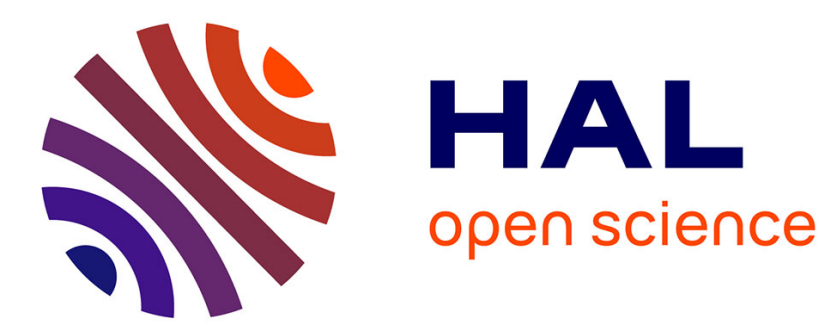

\title{
Le déploiement d'un pilotage stratégique des coûts dans les services informatiques de deux groupes internationaux : perspective instrumentale et analyse comparative
}

Grégory Wegmann

\section{To cite this version:}

Grégory Wegmann. Le déploiement d'un pilotage stratégique des coûts dans les services informatiques de deux groupes internationaux: perspective instrumentale et analyse comparative. Revue management \& avenir, 2011, 44, pp.78-96. hal-00624488

\section{HAL Id: hal-00624488 \\ https://hal.science/hal-00624488}

Submitted on 18 Sep 2011

HAL is a multi-disciplinary open access archive for the deposit and dissemination of scientific research documents, whether they are published or not. The documents may come from teaching and research institutions in France or abroad, or from public or private research centers.
L'archive ouverte pluridisciplinaire HAL, est destinée au dépôt et à la diffusion de documents scientifiques de niveau recherche, publiés ou non, émanant des établissements d'enseignement et de recherche français ou étrangers, des laboratoires publics ou privés. 


\title{
Le déploiement d'un pilotage stratégique des coûts dans les services informatiques de deux groupes internationaux : perspective instrumentale et analyse comparative
} Development of a strategic cost management in the
information systems departments of two international
groups: Instrumental and comparative studies

\author{
Grégory WEGMANN \\ Maître de Conférences (HDR) \\ Université de Bourgogne, IAE de Dijon, LEG/Fargo, UMR CNRS 5118 \\ Gregory.Wegmann@u-bourgogne.fr
}

Résumé : Cet article analyse le pilotage stratégique des coûts dans une perspective instrumentale et au travers de deux études de cas. Il s'agit de montrer en quoi les dispositifs de pilotage à base d'activités s'inscrivent dans une optique stratégique du contrôle de gestion et de tester si l'ABC est un outil pertinent de pilotage stratégique des coûts. La première partie revient sur les développements en pilotage stratégique des coûts et leurs liens avec les évolutions de l'ABC. La seconde partie présente une grille de lecture des motifs d'usage des dispositifs à base d'activités et explore le potentiel stratégique de plusieurs applications. Dans une troisième partie, nous confrontons nos développements théoriques et instrumentaux à la réalité du terrain au travers de deux études de cas.

Mots clés : Pilotage stratégique des coûts, Dispositifs à base d'activités, Etudes de cas

\begin{abstract}
This article analyses the strategic cost management stream with an instrumental point of view and two case studies. We try to show in what extend the Activity-based Costing developments could be included in a strategic approach of the management accounting and to test if the $\mathrm{ABC}$ is a relevant tool to drive the strategy. The first part synthesizes the strategic cost management developments which try to improve the Activity-based Costing method. In the first part, we describe them using the Strategic Management Accounting stream, with a link with cost management and $\mathrm{ABC}$. The second part exposes a taxonomy of the reasons why using the $\mathrm{ABC}$ method. In a third part, we confront our developments to the field reality with two case studies.
\end{abstract}

Key words: Strategic cost management, Activity-based Costing, Case studies 


\title{
Le déploiement d'un pilotage stratégique des coûts dans les services informatiques de deux groupes internationaux : perspective instrumentale et analyse comparative
}

\section{Development of a strategic cost management in the information systems departments of two international groups: Instrumental and comparative studies}

Résumé : Cet article analyse le pilotage stratégique des coûts dans une perspective instrumentale et au travers de deux études de cas. Il s'agit de montrer en quoi les dispositifs de pilotage à base d'activités s'inscrivent dans une optique stratégique du contrôle de gestion et de tester si l'ABC est un outil pertinent de pilotage stratégique des coûts. La première partie revient sur les développements en pilotage stratégique des coûts et ses liens avec les évolutions de l'ABC. La seconde partie présente une grille de lecture des motifs d'usage des dispositifs à base d'activités et explore le potentiel stratégique de plusieurs applications. Dans une troisième partie, nous confrontons nos développements théoriques et instrumentaux à la réalité du terrain au travers de deux études de cas.

Mots clés : Pilotage stratégique des coûts, Dispositifs à base d'activités, Etudes de cas

\begin{abstract}
This article analyses the strategic cost management stream with an instrumental point of view and two case studies. We try to show in what extend the Activity-based Costing developments could be included in a strategic approach of the management accounting and to test if the $\mathrm{ABC}$ is a relevant tool to drive the strategy. The first part synthesizes the strategic cost management developments which try to improve the Activity-based Costing method. In the first part, we describe them using the Strategic Management Accounting stream, with a link with cost management and $\mathrm{ABC}$. The second part exposes a taxonomy of the reasons why using the $\mathrm{ABC}$ method. In a third part, we confront our developments to the field reality with two case studies.
\end{abstract}

Key words: Strategic cost management, Activity-based Costing, Case studies

Une des questions centrales des recherches en contrôle de gestion et en management stratégique, depuis notamment les travaux de Johnson et Kaplan (1987), tient à la façon de mieux articuler le pilotage stratégique au pilotage opérationnel. Les managers des grandes entreprises, soucieux d'être plus réactifs, souhaitent des dispositifs de pilotage qui permettent d'anticiper les évolutions de l'environnement concurrentiel. Dans cet article, nous nous situons dans le champ du pilotage stratégique des coûts et dans une perspective instrumentale. Nous montrons que des outils de contrôle de gestion, basés sur la technique comptable de gestion à base d'activités (méthode $\mathrm{ABC}$, Activity-based Costing), peuvent s'inscrire dans une perspective stratégique du contrôle de gestion.

Notre raisonnement nous conduit dans un premier temps à expliquer que les approches à base d'activités peuvent constituer une forme a priori pertinente de pilotage stratégique des coûts. Les caractéristiques intrinsèques de l'ABC sont susceptibles de permettre un décryptage sophistiqué de l'architecture organisationnelle, propice à faciliter une lecture des liens entre le pilotage opérationnel et le pilotage stratégique. Dans un second temps, nous testons ces considérations en nous appuyant sur la littérature, puis sur deux études de cas. 
C'est pourquoi la première partie revient sur le concept de pilotage stratégique des coûts qui constitue une formalisation de l'articulation entre le contrôle de gestion et le management stratégique, en lien avec l'ABC, et la seconde partie présente une grille de lecture des motifs d'usage de la comptabilité de gestion à base d'activités et explore le potentiel stratégique des applications $\mathrm{ABC}$ étudiées. En quoi les développements sur l'ABC (ABC orienté clients, time-driven $\mathrm{ABC}, \ldots)$ témoignent-ils du potentiel stratégique de l'outil ? Dans une troisième partie, nous confrontons nos développements à la réalité du terrain au travers de deux études de cas. Nous avons étudié le développement de l'ABC dans les services informatiques de deux groupes, l'un industriel, l'autre bancaire. Les modèles $\mathrm{ABC}$ étudiés dans ces deux groupes constituent-ils des outils de pilotage stratégique des coûts?

\section{Le pilotage stratégique des coûts}

Dans cette partie, nous développons le concept de pilotage stratégique des coûts, en lien avec la méthode $\mathrm{ABC}$. Les premiers développements proposés nous permettent par la suite de nous demander si la méthode $\mathrm{ABC}$ est susceptible de constituer un outil de pilotage stratégique des coûts.

\subsection{La notion de pilotage stratégique des coûts}

Historiquement, le contrôle de gestion se décline en trois processus : le contrôle stratégique, le contrôle de gestion et le contrôle opérationnel (Anthony, 1965). La coordination entre ces processus est souvent décrite comme problématique (Johnson et Kaplan, 1987). Le pilotage stratégique des coûts ${ }^{1}$ renvoie à un concept et à des pratiques visant à résoudre les questions liées à la coordination entre ces processus.

La notion de pilotage stratégique des coûts est apparue au début des années 1980 (Simmonds, 1981) et a été approfondie au fil du temps (Lorino, 1991 ; Tomkins et Carr, 1996). C'est une notion instrumentale, puisque les différents développements étudiés aboutissent à des modèles qui articulent les dimensions stratégique, marketing et opérationnelle de la prise de décision. Simmonds (1981) et Bromwich (1990) proposent par exemple de déployer dans l'entreprise des mesures qualitatives tournées vers l'extérieur (du type taux de satisfaction des clients) selon trois dimensions : les produits, les clients et l'environnement concurrentiel. En nous appuyant sur Teller (1999, p. 40-41), trois approches du pilotage stratégique des coûts peuvent être discriminées.

- Une approche minimaliste où le contrôle de gestion est perçu comme un mode de contrôle de la mise en œuvre de la stratégie. Il s'agit d'une vision a posteriori du contrôle de la stratégie qui ne nécessite pas une étude et une déclinaison approfondies des déterminants de la stratégie. Il n'est pas nécessaire de retravailler l'architecture de l'organisation; ce mode de pilotage stratégique des coûts ne nécessite pas un accroissement des connaissances.

- Une approche médiane où le contrôle de gestion est appréhendé comme un mode de validation des hypothèses stratégiques. Cette fois, il s'agit d'une vision plus en amont du contrôle de la stratégie qui nécessite de décrypter les hypothèses stratégiques. Ces dernières constituent des facteurs clés de succès que le contrôle de gestion doit décliner le long de la chaîne de valeur de l'entreprise sous la forme de facteurs déterminants des coûts.

- Une approche élargie où le contrôle de gestion est considéré comme un élément constitutif de la formulation et de la mise en œuvre de la stratégie. Le contrôle est interactif (Simons,

\footnotetext{
${ }^{1}$ Par «pilotage stratégique des coûts », nous englobons différentes expressions comme «contrôle stratégique » (Bromwich, 1990) et «gestion stratégique des coûts » (Shank et Govindarajan, 1989) ou contrôle de gestion stratégique.
} 
1995 ; Le Moigne, 1996) et les nouvelles stratégies émergent au fil du temps. En fonction des contextes stratégiques et de leurs incertitudes, les dirigeants utilisent des outils de contrôle de gestion pour articuler le stratégique à l'opérationnel et faire émerger de nouvelles opportunités stratégiques.

Pour justifier l'intérêt au déploiement de dispositifs de pilotage stratégique des coûts, nous insistons sur le fait que l'environnement concurrentiel est devenu de plus en plus complexe et turbulent à partir de la fin des années 1970 et que le phénomène s'est accentué au cours des années 1980, et surtout sur le fait que les architectures organisationnelles des entreprises se sont complexifiées (Johnson et Kaplan, 1987). C'est pourquoi il est intéressant de disposer d'outils de contrôle de gestion susceptibles de décrypter cet environnement et ces structures d'une façon dynamique. Bouquin écrit (2006, p. 337) que dans ce contexte complexe et turbulent, «...la comptabilité de gestion et la gestion stratégique sont alors vouées à former un tout....» La méthode $\mathrm{ABC}$ peut-elle constituer une part essentielle d'un dispositif de pilotage stratégique des coûts en permettant de piloter chaque étape du déploiement d'une stratégie et le suivi de la réalisation des objectifs stratégiques, en validant les hypothèses stratégiques et éventuellement en stimulant de nouvelles orientations ?

L'enquête menée par Hoffjan et Wömpener (2006, p. 248) met en évidence le fait que les outils de gestion stratégique des coûts sont aujourd'hui nombreux (calculs de coûts orientés sur l'analyse des clients, coûts cibles, méthode $\mathrm{ABC}$, coûts prévisionnels, ...) Dans cet article, nous allons déterminer dans quelle mesure la méthode $\mathrm{ABC}$ constitue un outil de pilotage stratégique des coûts.

\subsection{La méthode $\mathrm{ABC}$ comme dispositif de pilotage stratégique des coûts}

La comptabilité de gestion à base d'activités (ABC, Activity-based Costing, Cooper et Kaplan, 1988) a été promue au milieu des années 1980 par des travaux effectués aux Etats-Unis, dans le cadre du Cam-i ${ }^{2}$, ainsi que par des universitaires de Harvard. Les évolutions de l'environnement, des technologies et des systèmes productifs avaient alors rendu plus sensible le problème de l'homogénéité des coûts. Aussi, les techniques en coûts complets traditionnelles entraînaient dans bien des cas des distorsions lors de l'allocation des coûts indirects aux objets de coûts. D'où l'idée de répartir ces coûts indirects dans des ensembles plus fins, les activités, au sein desquelles les coûts seraient davantage homogènes et en utilisant des clés de répartition plus fines que les unités d'œuvre, les inducteurs.

Le pilotage stratégique des coûts, dans ses versions médiane et élargie, implique d'adopter une approche processus de la comptabilité de gestion car l'entreprise est modélisée comme un réseau de structures et de projets transversaux constitués selon les impératifs stratégiques de l'entreprise. Dans ce contexte, les structures organisationnelles sont plus complexes à déchiffrer et la méthode $\mathrm{ABC}$ constitue un outil susceptible d'analyser ces structures. Les inducteurs, facteurs explicatifs des coûts, peuvent permettre d'appréhender les relations entre les processus et de relier les consommations de ressources aux projets stratégiques ainsi qu'aux compétences nécessaires pour les réaliser. La structure de la méthode $\mathrm{ABC}$ et le rôle pivot joué par les inducteurs, permet de comprendre la dimension a priori stratégique de la méthode. Ces inducteurs décrivent les processus de consommation des ressources et permettent de remonter causalement la chaîne de valeur de l'entreprise et ainsi d'envisager d'établir une articulation entre le pilotage stratégique et opérationnel. D'où l'émergence du Management à base d'Activités (ABM, Activity-based Management), dans le prolongement de $1^{\prime} A B C^{3}$.

\footnotetext{
${ }^{2}$ Consortium for Advanced Manufacturing International.

${ }^{3}$ Dans cet article, nous faisons un usage élargi du terme $\mathrm{ABC}$, en y incluant une dimension managériale. En ce sens, il n'y a pas de différence entre l'ABC et l'ABM.
} 
La dimension stratégique accompagne de fait l'ABC dès son émergence à la fin des années 1980. Ainsi, Jones et Dugdale (2002) mettent en évidence les liaisons entre l'ABC et l'école du pilotage stratégique des coûts, ainsi que Shank et Govindarajan (1989). D'une façon similaire, l'un des premiers ouvrages français sur l'ABC (Lorino, 1991) énonce l'idée d'une connexion entre la méthode et le pilotage stratégique stratégique. C'est dans cet esprit que Shank et Govindarajan se fondent sur les concepts de cycle de vie et de chaîne de valeur pour préconiser d'intégrer les dimensions clients et fournisseurs dans les calculs de coûts à base d'activités. En France, sur la base d'une réflexion théorique et de deux études de cas, Chauvey et Naro (2004) décrivent le potentiel de l'ABC comme outil de gestion stratégique des coûts (d'analyse des stratégies déployés et de dévoilement des stratégies émergentes).

Si l'on considère que le contrôle de gestion constitue un des composants de l'architecture d'une organisation (Jensen et Meckling, 1992), et si l'on se place dans une perspective contractuelle (théories de l'agence et des coûts de transaction : Brickley et al., 1997), le contrôle de gestion a une triple mission : contribuer à réduire les conflits d'intérêts, aider à une allocation optimale des ressources et assurer la convergence des buts de façon à garantir la cohérence interne de la firme. Cette triple mission consiste en synthèse à contribuer à décliner les objectifs stratégiques de l'entreprise dans ses différentes composantes (logique de l'alignement stratégique), ce qui correspond à la version restreinte du pilotage stratégique des coûts, les objectifs n'étant pas questionnés. Or la méthode $\mathrm{ABC}$ incite à discuter les objectifs stratégiques de l'entreprise car les inducteurs constituent des variables qui permettent de valider ou d'invalider des corrélations entre des choix stratégiques (investissements dans la formation par exemple) et leurs effets (évolution des niveaux de productivité), ce qui peut conduire à réorienter les choix stratégiques le cas échéant. Autrement dit la méthode $\mathrm{ABC}$ s'inscrit dans les versions médianes et élargies du contrôle de gestion, ce qui implique un pilotage participatif car les acteurs du processus de contrôle doivent développer une connaissance approfondie et dynamique des déterminants des coûts de l'entreprise. C'est pourquoi une approche stratégique de l'ABC incite à épouser une logique fondée sur les connaissances. La théorie de l'apprentissage organisationnel (Argyris et Schön, 1978) et celle fondée sur les compétences clés (Hamel et Prahalad, 1990) constituent des grilles de lectures complémentaires pour analyser les dispositifs de pilotage stratégique et l'ABC. La création de valeur est alors analysée sous l'angle de l'accroissement des connaissances au sein de la firme. Ainsi, un outil de pilotage stratégique des coûts est à la fois un moyen de mieux allouer les ressources (perspective contractuelle) et de faciliter le développement des compétences (perspective cognitive).

Mais la littérature a mis en évidence à de nombreuses reprises les limites de l'ABC et remis en question son potentiel stratégique.

\subsection{Les limites de la méthode $\mathrm{ABC}$}

Les limites de l'ABC ont en effet fait l'objet de nombreuses critiques dans les pays AngloSaxons (Anderson and Young, 1999; Datar and Gupta, 1994; Foster and Swenson, 1997; Malmi, 1997). En France, Bouquin (2006) et Chatelain et Sponem (2007) montrent que les principes de l'ABC sont très proches de ceux des sections homogènes, la méthode rencontrant ainsi des limites du même ordre. En se fondant sur les systèmes traditionnels comptables, l'ABC ne permet pas d'appréhender l'ensemble des coûts et performances et en particulier les coûts humains des activités ${ }^{4}$. Berland et Gervais (2008) expliquent que les travaux français sur l'ABC ont été nombreux et qu'ils soulèvent de nombreuses interrogations notamment sur les paramètres de conception de la méthode (Mévellec, 2005).

\footnotetext{
${ }^{4}$ Voir les développements de Savall et Zardet (2010) relatifs aux coûts et performances cachées.
} 
Plus précisément, Mévellec (2005) et Gervais et Lesage (2006), reprenant et complétant les auteurs Anglo-Saxons précités, expliquent que l'ABC ne parvient qu'imparfaitement à résoudre plusieurs types d'erreurs : erreurs d'agrégation (des ensembles de ressources), de mesure (coûts d'une activité et nombre d'inducteurs), de spécification et d'imputation des charges fixes (non prise en compte de la sous-utilisation des capacités de production). Les systèmes $\mathrm{ABC}$ s'avèrent dans certains cas d'une complexité extrême qui les éloignent de la mission d'aide à la prise de décision. Dans d'autres cas, l'on procède à des simplifications abusives. La méthodologie a du mal à s'adapter à l'univers des services et les dimensions marketing et commerciales seraient négligées.

Cependant, à côté de cette littérature critique, nous relevons des travaux qui mettent en évidence le potentiel stratégique de l'ABC. C'est pourquoi nous décrivons à présent les dimensions d'usage de l'ABC, en liaison avec les développements autour de l'ABC (partie 2), puis en analysant deux études de cas (partie 3).

\section{Les dimensions d'usage des comptabilités à base d'activités et leur potentiel stratégique}

Dans cette partie, nous dressons tout d'abord un état de l'art des fonctionnalités possibles de la méthode $\mathrm{ABC}$ que nous intitulons «dimensions d'usage». Puis nous approfondissons certaines d'entre-elles en nous appuyant sur des développements relatifs à l'ABC. Notre question centrale est: en quoi certains développements sur l'ABC ( $\mathrm{ABC}$ orienté parties prenantes, time-driven $\mathrm{ABC}, \ldots$ ) expriment-ils son potentiel stratégique ? L'objectif est de savoir si nos réflexions théoriques sur le caractère a priori stratégique de l'ABC peuvent être validées par des développements instrumentaux.

\subsection{Les dimensions d'usage de la méthode $\mathrm{ABC}$}

Dans le tableau 1, nous proposons une typologie des dimensions d'usage de la méthode ABC. Nous nous sommes appuyés sur les travaux de Mévellec (2005) concernant les paramètres de conception des systèmes de calculs de coûts ainsi que sur les développements relatifs aux outils présentés dans la suite de l'article.

Comme le montre Bouquin (2006), l'origine de la méthode ABC s'inscrit dans une réflexion sur le niveau d'analyse pertinent des coûts. Est-il pertinent d'analyser les coûts au niveau des produits (ou des services, clients, ...), des processus, des centres d'analyse, des activités, des tâches, etc ? Cela dépend de considérations techniques (notion d'ensemble de coûts homogènes) et managériales (que voulons nous piloter ?) En synthèse, les démarches à base d'activités poursuivent un triple objectif: améliorer la traçabilité des coûts, modéliser les variables clés de la performance (facteurs déterminants des coûts, inducteurs) et développer des systèmes de pilotage des coûts stratégiques et transversaux.

Dans le tableau 1, nous distinguons quatre dimensions d'usage de l'ABC. Les dispositifs recensés dans la littérature s'appuient, à des degrés divers, sur l'une ou plusieurs de ces dimensions. Celles-ci ne sont donc pas mutuellement exclusives. La première dimension renvoie à une approche extravertie et partenariale de l'ABC. Elle s'inscrit donc dans le champ de la théorie des parties prenantes (Freeman, 1984) où l'on considère que leur prise en compte a un impact positif sur la performance. Bouquin (2006, p. 228-229) explique ce phénomène en prenant l'exemple du développement outre-atlantique du Customer Relationship Management (management de la relation clients, Horngren, 2005) qui vise à optimiser les processus de gestion de la clientèle. La seconde dimension se réfère à une vision prospective de l'ABC. Les dispositifs mentionnés dans le tableau 1 intègrent, à des degrés divers et selon des modalités différentes, une analyse prévisionnelle des coûts (Antos et Brimson, 1999, Horvath, 1998 et Cokins 2002). 
Tableau 1 - Les dimensions d'usage de la méthode ABC

\begin{tabular}{|c|c|}
\hline Dimensions d'usage de la méthode $\mathrm{ABC}$ & Exemples de dispositifs proposés \\
\hline $\begin{array}{l}\text { Dimension 1: les partenaires } \\
\text { Elargissement spatial du } \\
\text { périmètre d'analyse des coûts : } \\
\text { aux clients, fournisseurs, circuits de } \\
\text { distribution et autres parties prenantes }\end{array}$ & $\begin{array}{l}\text { Analyse ABC des clients profitables } \\
\text { Benchmarking externe } \\
\text { Gestion interorganisationnelle des coûts } \\
\text { Coûts cibles à base d'activités }\end{array}$ \\
\hline $\begin{array}{c}\text { Dimension 2 : élargissement temporel } \\
\text { du périmètre des coûts : } \\
\text { au-delà de l'exercice comptable, } \\
\text { sur un cycle de vie, sur un projet }\end{array}$ & $\begin{array}{l}\text { Planification et budgets à base d'activités } \\
\text { Gestion sans budgets } \\
\text { Coûts cibles à base d'activités }\end{array}$ \\
\hline $\begin{array}{l}\text { Dimension 3 : complexification } \\
\text { du modèle d'analyse } \\
\text { Rafinement de la phase d'allocation des ressources, } \\
\text { variété des facteurs déterminants des coûts } \\
\text { et fiabilisation des modèles }\end{array}$ & $\begin{array}{c}\text { Comptabilité de gestion à base de consommation de ressources } \\
\text { méthodes statistiques de fiabilisation des données }\end{array}$ \\
\hline $\begin{array}{l}\text { Dimension } 4 \text { : simplification } \\
\text { du modèle d'analyse } \\
\text { Simplification de la maille d'analyse } \\
\text { réduction du nombre de variables } \\
\text { d'analyse des coûts }\end{array}$ & $\begin{array}{l}\text { ABC à base d'équivalent temps } \\
\text { Comptabilité de gestion à base de processus } \\
\text { Méthode des coûts caractéristiques }\end{array}$ \\
\hline
\end{tabular}

La troisième dimension vise à caractériser les dispositifs $\mathrm{ABC}$ qui se sont complexifiés. Tenant compte des nombreuses erreurs relevées à l'occasion de mises en place de systèmes $\mathrm{ABC}$, des auteurs développent des recherches relatives à une complexification de la maille d'analyse des coûts, à une tentative de renforcement de la rationalisation de la phase d'allocation des ressources (Keys et van der Merwe, 2002) ou encore à des méthodes statistiques visant à fiabiliser les calculs à base d'activités (Thomas et Gervais, 2008). Lorsque l'on observe par exemple une grande variété de ressources consommées dans un processus, la méthode $\mathrm{ABC}$ peine parfois à allouer rationnellement ces ressources aux activités (erreurs d'agrégation). La quatrième dimension de notre typologie s'intéresse cette fois à une simplification des modèles ABC (Brimson, 1998, Johnson et Bröms, 2002 ; Kaplan et Anderson, 2007) et à un regroupement des activités dans des processus clés. Il faut cependant imaginer un continuum entre les troisième et quatrième dimensions, un système $\mathrm{ABC}$ pouvant combiner des processus complexes à des processus plus simplifiés. Reprenons à présent ces quatre dimensions et inscrivons-y les développements sur l'ABC recensés en nous interrogeant sur leur potentiel stratégique.

\subsection{Approfondissements sur l'orientation stratégique des modèles à base d'activités}

Les dispositifs à base d'activités qui insistent sur la première dimension (tableau 1) tentent d'élargir le périmètre d'analyse des coûts aux clients, circuits de distribution, fournisseurs et autres parties prenantes. Une cartographie des activités peut contribuer à l'analyse des 
interfaces entre une entreprise et ses clients, fournisseurs et autres parties prenantes. Des modèles ABC orientés clients (CPA, Customer Profitability Analysis, analyse des clients profitables, Cooper et Kaplan, 1999, p. 352 s.), fournisseurs, sous-traitants et partenaires (Interorganizational Cost Management, IOCM, management inter-organisationnel des coûts) ont été développés en ce sens. Lebas (1999) explique que très rapidement les travaux sur l'ABC ont intégré la dimension clients. Mévellec (2005) décrit différentes versions d'ABC où l'analyse des coûts est orientée sur les clients (ABC011 p. 228-239, ABC101 p. 250-259 et ABC111 p. 270-279). Kuchta et Troska (2007, p. 18) expliquent que l'ABC est un bon moyen d'établir des profitabilités comparées de clients et de cibler les activités déterminantes dans l'explication de ces profitabilités et sur lesquelles il convient de faire porter des efforts. Caglio et Ditillo (2008) proposent une synthèse du «contrôle de gestion inter-organisationnel », thème également traité dans les revues françaises (Berland et Gervais, 2008, p. 131). Cooper et Slagmulder (2004) inscrivent l'ABC dans ce thème avec le management interorganisationnel des coûts qui résulte d'observations effectuées sur des entreprises japonaises. L'ABC permet de décrypter la chaîne de valeur commune aux entreprises concernées par un management inter-organisationnel et d'initier un processus $d$ ' « investigation organisationnelle des coûts ».

Concernant la seconde dimension, des modèles tels que les budgets à base d'activités (Antos et Brimson, 1999), la planification à base d'activités, les coûts cibles à base d'activités (Cokins 2002, Horvath, 1998) et la gestion sans budgets (Cam-i), s'appuient sur une approche prospective de l'ABC. On pourrait penser que les dispositifs qui s'inscrivent dans la troisième dimension des motifs d'usage n'ont pas une vocation stratégique majeure. Cependant, on peut considérer qu'un dispositif tel que la comptabilité à base de consommation de ressources (RCA, Resource Consumption Accounting, Keys et van der Merwe, 2002), en tentant d'identifier au mieux la cause des coûts, constitue une tentative pour remonter la chaîne des coûts jusqu'aux déterminants stratégiques. La RCA revient à complexifier l'ABC puisque se combinent deux phases d'allocation, des ressources aux pools de ressources et des ressources aux activités, et que les éléments variables des éléments fixes sont systématiquement dissociés. Elle peut ainsi permettre de corriger les erreurs de spécification lorsque le volume des charges d'une activité dépend à la fois de l'inducteur choisi et du volume d'autres inducteurs, ce qui ensuite facilite l'analyse de la chaîne des coûts et au final la lecture de la stratégie. Dans le même esprit, Thomas et Gervais (2008) développent une méthode pour tester l'homogénéité des activités et la pertinence des inducteurs retenus. Ils proposent, en s'appuyant sur une étude de cas, de mener une analyse en composantes principales (ACP) combinée à une analyse factorielle. Cette démarche permet d'aboutir à la mise en évidence de « méta-inducteurs » représentatifs pour chacun d'entre eux d'un panier d'inducteurs. C'est une façon d'articuler les niveaux stratégique et opérationnel et en cela, faire œuvre de pilotage stratégique des coûts.

Concernant la quatrième dimension, dans certaines situations, le degré de complexité de la phase d'allocation des ressources est faible et un $\mathrm{ABC}$ simplifié peut se révéler pertinent. Des auteurs suggèrent ainsi de modéliser un nombre limité d'activités et notamment d'allouer les coûts à des «méta-activités » ou à des processus (Horngren et al., 2005, p. 594 s.). Johnson et Bröms (2002) développent le Management by Means (MBM) qui est un ABC simplifié, les activités étant regroupées en trois processus majeurs, ceux des activités courantes, du développement des activités futures et des activités de soutien. En outre le MBM utilise la ligne de commande comme objet de coût. Kaplan et Anderson (2007) développent le TimeDriven $\mathrm{ABC}, 1$ 'ABC à base d'équivalents temps. Le principe général de la méthode est de traduire les coûts des différents inducteurs en équivalents temps (heures de travail), révisables lorsque les conditions de production sont modifiées (logique des coûts préétablis, Bouquin, 
2006, p. 306 $)^{5}$. L'hypothèse forte de la technique est qu'il est possible de modéliser le comportement des coûts sur la base des temps passés à réaliser les tâches.

Les travaux présentés dans cette partie montrent, malgré les limites relevées, le potentiel stratégique de l'ABC sur certains aspects. Nous allons à présent confronter nos propositions à la réalité du terrain en analysant deux études de cas et en questionnant le caractère stratégique des ABC déployés.

\section{Etudes de cas}

Dans cette partie, nous présentons et confrontons deux expériences de déploiement de l'ABC. Comme l'explique Sabbah (2009), «le pilotage des coûts informatiques a souvent été le "parent pauvre » du contrôle de gestion ", d'où l'intérêt de montrer des déploiements de l'ABC dans ce domaine. Ces deux expériences se déroulent au sein des services informatiques de deux grands groupes. Le premier est un géant international de l'industrie que nous intitulons Groupe 1 et le second est une grande banque française (Groupe 2). Nous avons jugé intéressant d'étudier des services immatériels à haute valeur ajoutée (liés au déploiement de systèmes d'information). Nous analysons ces études de cas à l'aune de nos développements sur le pilotage stratégique des coûts.

\subsection{Méthodologie de l'étude et collecte des données}

Nous avons suivi ces expériences entre début 2007 et fin 2009 en réalisant des interviews et en étudiant les différentes documentations mises à notre disposition. Il ne s'agissait pas d'observations participantes mais de plusieurs entretiens menés avec les deux chefs de projet, les membres du groupe projet et les consultants externes.

Une première vague d'entretiens a été menée au début des projets (été 2007), une seconde au printemps 2008, une troisième au printemps 2009 et une dernière à la fin des projets. Il s'agissait d'entretiens semi-directifs. Concrètement, tous les membres des services concernés dans les deux entreprises ont été interviewés à trois reprises, ce qui au final représente une cinquantaine d'entretiens d'environ $1 / 2$ heure chacun. Des entretiens ont également été menés avec des contrôleurs de gestion d'autres départements des entreprises concernées. Les contacts avec les chefs de projet étaient plus suivis (échanges d'emails, conversations téléphoniques, rendez-vous, ...) La grille d'entretien utilisée, très ouverte, définit des thèmes principaux et permet aux échanges d'évoluer librement sur la base de ces thèmes. Il s'agissait de comprendre la structure organisationnelle des entreprises et services étudiés, de connaître les systèmes historiquement déployés, d'identifier les motivations à l'origine des projets $\mathrm{ABC}$, de comprendre le cheminement utilisé pour construire les nouveaux outils, les objectifs qui leur ont été assignés et d'appréhender les difficultés et les limites rencontrées.

\subsection{Le déploiement de l'ABC au sein du Groupe 1 : un pilotage simplifié et transversal}

Nous avons étudié la mise en place d'un système $\mathrm{ABC}$ au sein d'une division autonome ${ }^{6}$ (centre de profits) qui délivre une gamme étoffée de services informatiques (fourniture de matériels, actions de formation, configuration de réseaux, etc.) aux clients internes (les autres divisions du groupe) et à des clients externes. Le système de gestion d'Infotech reposait sur une comptabilité analytique basée sur un découpage en centres de coûts et la détermination de coûts de revient par produit. Mais ce système ne fournissait pas une information suffisamment précise pour aider les managers à construire une gamme tarifaire qui tienne compte des coûts

\footnotetext{
${ }^{5}$ Le TDABC est discuté par la communauté des chercheurs (La Villarmois (de) et Levant, 2007, Gervais et al., 2010). Gervais et al. mettent en évidence des incertitudes conceptuelles et méthodologiques sur notamment le choix entre coûts standards et coûts réels (p. 5-6), et les calculs de coûts de sous-activité (p. 7-8).

${ }^{6}$ Nous nommons la division informatique du groupe «Infotech ».
} 
générés par chaque client en fonction de sa demande. Ce système ne permettait pas de relier l'analyse des coûts à la stratégie. Le contrôleur de gestion de la division, convaincu que l'ABC pourrait constituer un outil pertinent de pilotage stratégique des coûts, reçu mission de sa direction de mener le déploiement d'un $\mathrm{ABC}$. La figure 1 décrit l'organisation du département étudié.

\section{Figure 1}

Organisation de la division Infotech

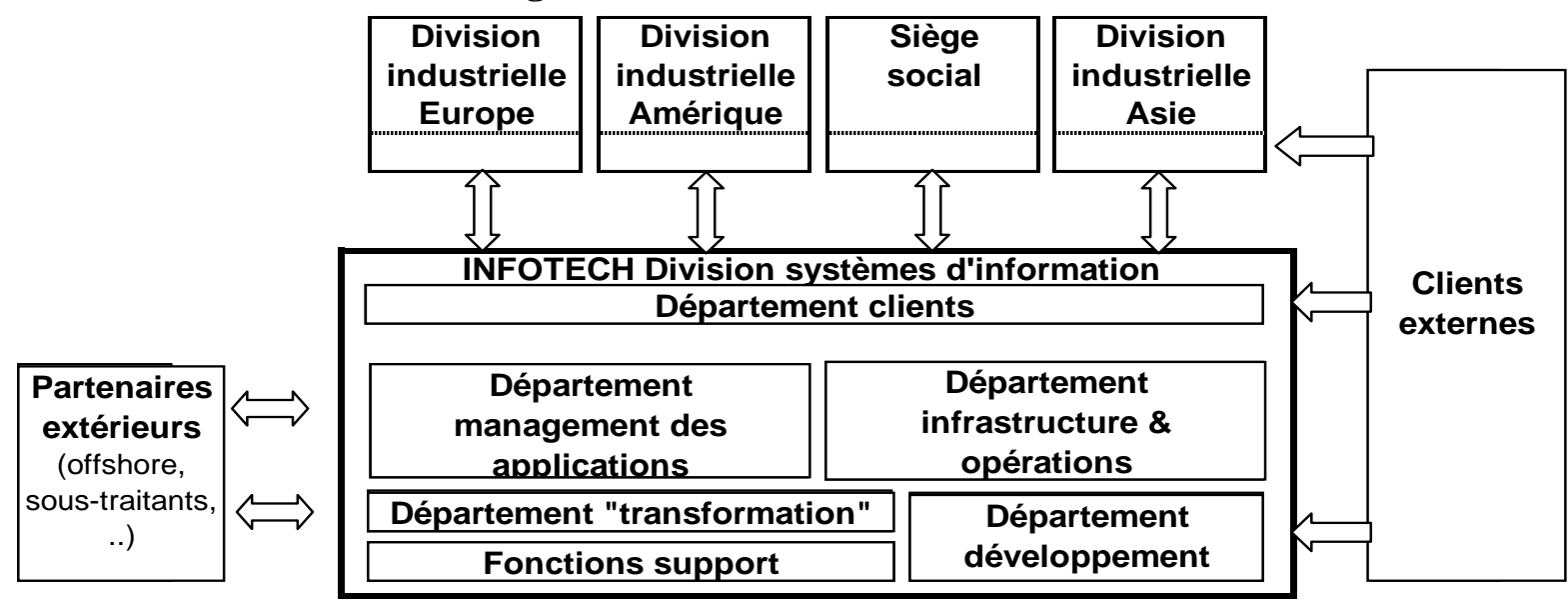

La première étape a consisté en un diagnostique de la situation initiale, une planification du projet et la constitution du groupe projet. Ensuite, ce groupe projet a identifié les activités en interviewant les managers. Une première liste d'activités est élaborée, puis affinée grâce à des entretiens individuels et collectifs et des retours d'expérience avec tout le personnel (approche participative). Ces activités sont déterminées sur la base d'une logique processus, c'est-à-dire en référence aux objectifs stratégiques de la division, objectifs déclinés dans les processus clés de la structure. Puis il est procédé à une allocation des ressources aux activités avec l'utilisation notamment d'une «table des temps », les temps de travail des informaticiens constituant la clé de ventilation principale des ressources aux activités. Les compétences humaines de la division constituent un facteur prépondérant d'explication des coûts. Ensuite, le groupe projet détermine les inducteurs d'activité et calcul les coûts de revient. Enfin, il est procédé au calcul des profitabilités par types de services et par catégories de clients et à la validation du modèle, aux corrections et à l'analyse des résultats. Cette méthodologie a permis une allocation plus rationnelle des ressources aux activités, de gérer les activités comme des éléments de processus plus vastes, de piloter les coûts en fonction des services offerts aux clients, d'élaborer des mesures de benchmarking et au final de piloter la performance en partenariat avec les clients. L'équipe projet a pris conscience au fur et à mesure de l'avancée du projet du caractère stratégique de l'ABC ainsi déployé.

Sur la figure 2, nous proposons une extraction de plusieurs comptes analytiques, centres de coûts, propositions d'activités et catalogue de services. La liste d'activités proposée discrimine des activités récurrentes des activités projets. Les coûts de l'activité "management des imprimantes " sont par exemple alloués aux objets en fonction du nombre d'imprimantes délivrées à un client, les coûts de l'activité helpdesk en fonction du nombre d'appels d'un client. Ainsi, les clients constituent l'objet de coûts principal du modèle ABC. Cela signifie que l'outil décrit exploite la première dimension d'usage de la typologie présentée dans le tableau 1 en permettant une analyse des clients profitables. 
Figure 2

Extraction de comptes analytiques, centres de coûts, proposition d'activités et catalogue de services

Comptes

analytiques

Centres

de coûts

Activités

Objets de

coûts

Main d'œuvre
interne
externe (sous-traitance)
Dépenses informatiques
Hardware : location, crédit-bail
hardware : maintenance
Software : achat, location
Software : maintenance
Télécommunications
Autres
Déplacements
Locaux
Prestataires de services
Impôts et taxes
Amortissements
Hardware
Software
Autres
Revenus internes
France
Italie
Belgique
Espagne
Allemagne
Revenus externe

\begin{tabular}{|l|}
\hline Gestion des matériels \\
helpdesk \\
imprimantes \\
fixes et portables \\
distribution \& configuration \\
\\
Hébergement des applications \\
Serveurs NT, Unix, .... \\
Messagerie \\
Applications MVS \\
Applications Unix, SAP \\
Métrologie \\
Stockage \\
Datacenter \\
Services réseaux \\
Wan, lan, ... \\
Téléphonie \\
Internet \\
Administratif \\
Management interne \\
Gestion clients \\
\end{tabular}

\begin{tabular}{|c|c|c|c|}
\hline Inducteurs de & $\begin{array}{l}\text { Activités projets } \\
\text { (transversales) } \\
\text { Management projet } \\
\text { Analyse \& pré-étude } \\
\text { Construction } \\
\text { Développement } \\
\text { Activités communes } \\
\text { Achats } \\
\text { Organisation } \\
\text { Management global } \\
\text { Gestion financière }\end{array}$ & Inducteurs & $\begin{array}{l}\begin{array}{l}\text { Ordinateur fixe } \\
\text { conventionnel }\end{array} \\
\text { Portable } \\
\text { conventionnel } \\
\text { Outils sur mesure } \\
\text { Ecrans }\end{array}$ \\
\hline ressources & $\begin{array}{l}\text { Activités support } \\
\text { Support clients } \\
\text { Gestion des incidents } \\
\text { Coordination des demandes } \\
\text { Activités récurrentes } \\
\text { coordination des équipes } \\
\text { gestion des tâches } \\
\text { tests } \\
\text { gestion des utilisateurs } \\
\text { planification du travail } \\
\text { gestion des opérations } \\
\text { mesure des performances }\end{array}$ & d'activités & $\begin{array}{l}\text { Terminaux } \\
\text { Options } \\
\text { Imprimantes } \\
\text { Services } \\
\text { Messagerie } \\
\text { Progiciels }\end{array}$ \\
\hline
\end{tabular}

La plupart des activités sont transversales aux différents services de la division et beaucoup d'entre elles sont en lien direct avec les clients. Le modèle permet par exemple à un client de comprendre une facturation. La dimension inter-organisationnelle est présente puisqu'il s'agit de piloter des processus qui vont des fournisseurs aux clients. L'outil permet aussi de piloter les coûts en référence aux meilleures pratiques observées dans le groupe. On constate également qu'il s'agit d'un système $\mathrm{ABC}$ simplifié comportant un nombre réduit d'activités (une cinquantaine). Le modèle s'inscrit plutôt dans une logique processus avec des métaactivités pouvant traverser plusieurs centres de coûts. Le modèle ABC d'Infotech s'appuie donc également sur la quatrième dimension de notre typologie en proposant un modèle d'analyse simplifié, un nombre réduit de variables d'analyse des coûts et un inducteur de ressources «temps de travail» privilégié (logique TDBAC). La variété des inducteurs d'activités est en revanche plus importante avec des indicateurs retenus comme le «nombre d'incidents par client et type de service », le «nombre de requêtes clients », le «nombre de produits différents par client », le «nombre d'interventions chez un client », etc.

\subsection{Le déploiement de l'ABC au sein du Groupe 2 : un pilotage stratégique plus complexe}

Le projet $\mathrm{ABC}$ au sein du groupe 2 a été initié en 2004-2005, mais démarra réellement en 2007 et fut achevé fin 2009. Il s'agissait d'améliorer l'allocation des coûts afin de déterminer des coûts de revient fiables par prestation/produit/client. Le service en charge du projet se situe dans une organisation plus large qu'un contrôle de gestion classique. En effet, le responsable de la cellule manage une équipe chargée de gérer le suivi des projets informatiques et d'assurer la fiabilité de leur reporting. La vocation principale de l'outil est d'orienter les calculs de coûts vers le client final, interne au groupe, mais également vers des clients externes (particuliers ou entrepreneurs). Il sert ainsi à mieux négocier les tarifs avec les partenaires commerciaux. Dans ce cadre, l'informatique joue un rôle essentiel puisque par exemple, dans le calcul du coût d'un dossier de crédit, le coût informatique est très important. 
D'où une mission spécifique confiée à un contrôleur de gestion et consistant à adapter la méthodologie $\mathrm{ABC}$ aux spécificités des activités informatiques.

A la fin 2008, le projet en est à un travail sur la différenciation des crédits. Nos observations montrent que le système analytique par activités à élaborer est plus complexe que celui décrit dans l'étude de cas précédente. Il apparaît qu'il y a notamment une plus grande diversité dans les ressources consommées et que des inducteurs «temps» ne sont pas suffisants. En outre, beaucoup d'activités sont sous-traitées, ce qui pose le problème de la définition des métriques et la question d'une approche partenariale de la construction du modèle. Le groupe 2 se caractérise en effet par une forte externalisation des services, ce qui complexifie le système d'allocation des ressources sur les activités et les services. Afin de limiter les risques d'allocations non fiables et de subventionnements croisés, il est donc nécessaire d'intégrer fortement les partenaires au projet (logique de la gestion inter-organisationnelle des coûts, cf. première dimension d'usage du tableau 1).

Les coûts des ressources informatiques sont déversés sur les activités informatiques (administration / exploitation, réalisation de projets ...) grâce aux inducteurs de ressources (temps passés, $\mathrm{m}^{2}$, effectifs, détail de la facture production...) Les coûts des activités sont ensuite déversés sur les prestations fournies par l'informatique (mise à disposition d'applications crédits, monétiques...) via des inducteurs d'activité (puissance mainframe...), la fourniture de ces prestations consommant des activités. Les coûts des prestations sont ensuite déversés sur les clients de l'informatique (avec le détail par produit) via des inducteurs d'activité (instances par média, autorisations monétiques, nombre de dossiers validés, ...), les clients consommant des prestations. Le nombre d'activités est beaucoup plus important que pour le groupe 1 .

La figure 3 propose une cartographie du macro-modèle réalisée par le groupe 2 telle que formulée par l'équipe projet en charge de l'ABC.

Figure 3

\section{Cartographie du modèle du département informatique du groupe 2}

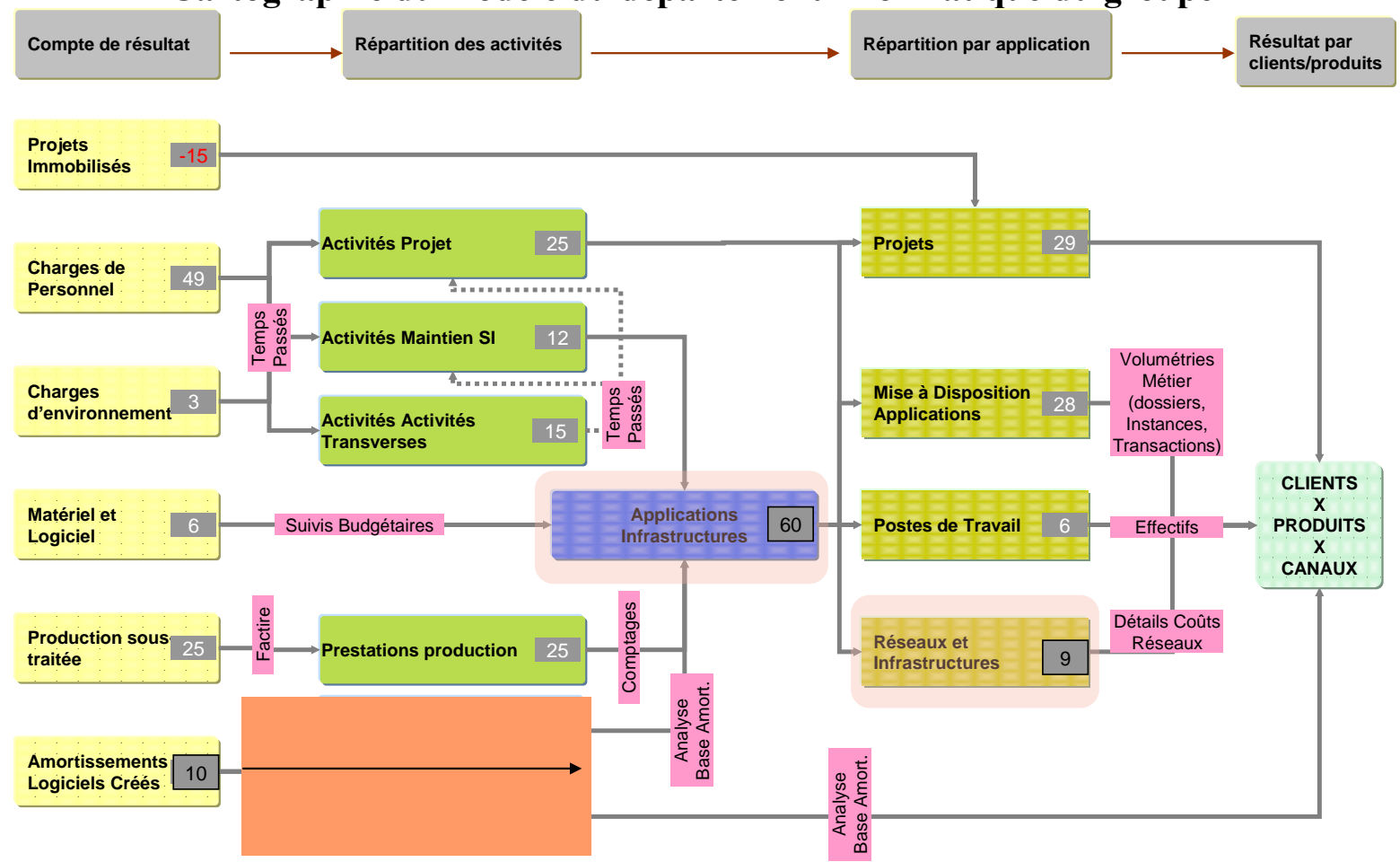

Montants exprimés en M€ (les chiffres sont fictifs) 
La démarche analytique aboutit à la mise en évidence de coûts de production différenciés et de coûts unitaires par famille de produits sur les environnements par pays et partenaires. L'analyse permet aussi de discriminer des coûts unitaires différenciés par dossier en cours, dossier validé, dossier " épargne » ou encore d'étudier les coûts du recouvrement. Cela a permis par exemple de fournir des explications sur la composition des coûts, et une renégociation auprès des partenaires/clients des tarifs pratiqués. Les managers du groupe 2 ont pu construire un argumentaire détaillé et rationnel de leurs offres. La dernière phase du projet s'est déroulée de fin juin 2009 à l'automne 2009 avec pour objectif de pouvoir analyser les résultats en $\mathrm{ABC}$ pour novembre (coûts de revient par client et par produit). Début juillet, un lancement a été mené avec les clients principaux, très impliqués dans le projet.

\subsection{Synthèse des deux études de cas et discussion}

En synthèse de la première étude de cas, nous considérons que le système d'Infotech est un $\mathrm{ABC}$ simplifié (quatrième dimension d'usage) qui combine plusieurs approches : clients, processus, fournisseurs (première dimension d'usage de notre typologie, perspective partenariale) et à base d'un inducteur temps. D'un point de vue organisationnel, il s'agit d'un modèle de déploiement participatif. Les concepteurs du dispositif à base d'activités ont besoin d'être proches du terrain et d'avoir une connaissance approfondie des métiers et des processus de l'entreprise. C'est pourquoi une approche bottom-up a été privilégiée, le contrôleur de gestion qui pilote le projet s'étant entouré d'un groupe de travail composé de salariés de la structure concernée. Cela a facilité aussi une diffusion des connaissances autour de la logique $\mathrm{ABC}$ (perspective cognitive). Concernant la seconde étude de cas, nous retrouvons la même dimension partenariale (clients internes et externes et négociations tarifaires avec les fournisseurs). Nous pouvons donc dire sur cet aspect que les deux études de cas mettent en évidence une orientation stratégique du pilotage car l'analyse de leurs objets de coûts et des inducteurs associés permettent de valider les hypothèses stratégiques formulées par les directions des deux divisions en termes de positionnement clients et de choix de fournisseurs et de partenaires. C'est pourquoi les deux modèles $\mathrm{ABC}$ illustrent plutôt l'approche médiane du pilotage stratégique des coûts développée dans la première partie. On peut aussi penser qu'ils puissent illustrer l'approche élargie du pilotage stratégique des coûts en ce sens que des calculs $\mathrm{ABC}$ par rapport à un prestataire de services pourraient par exemple remettre en cause un choix de sous-traitance. L'ABC dans les deux groupes a aussi permis de mener une réflexion sur la capacité des systèmes informatiques à héberger et servir les applications selon les volumes des dossiers clients. Des seuils de rentabilité ont été calculés pour optimiser les capacités informatiques par client de façon à rationaliser les coûts.

Cependant, la seconde expérience, à la différence de la première, s'inscrit dans une approche plus technicienne, avec la volonté d'aboutir à un modèle $\mathrm{ABC}$ plus sophistiqué qui puisse décrypter d'une façon assez fine les facteurs déterminants des coûts. De ce fait, la transparence des coûts est assez poussée, sans que l'on puisse dire que le second modèle consiste en une version $\mathrm{ABC}$ sophistiquée (cf. troisième dimension d'usage du tableau 1). En outre, des délais plus restreints dans le second cas, expliquent aussi que le nombre d'allersretours entre l'opérationnel informatique et l'équipe de conception ait été moins importants, d'où une approche moins participative. La direction exprime de fortes attentes, ce qui a aussi une incidence sur la gouvernance du projet.

Observons cependant plusieurs difficultés. Dans les deux cas, nos entretiens ont mis en évidence la difficulté à tracer certains coûts administratifs. Les coûts récurrents sont plus difficiles à tracer que les coûts projets et dans le secteur bancaire plus spécifiquement, on se heurte à beaucoup de coûts joints, en raison du caractère immatériel de nombreux processus. En outre, la maîtrise des temps déclarés est délicate. Les situations de gestion demeurent parfois délicates à interpréter. Des indicateurs peuvent être trompeurs et il faut les analyser 
avec une très grande prudence. Par exemple, un ratio « coût SI (systèmes d'information) /CA (chiffre d'affaires) » inférieur peut signifier une meilleure efficacité, mais peut aussi être la conséquence d'un sous-investissement. Ainsi, la mesure de la performance informatique reste un exercice complexe, même en s'appuyant sur l'outil ABC. Précisons aussi que les systèmes décrits ne tiennent pas compte des coûts et performances cachées (Savall et Zardet, 2010), ce qui peut conduire à des analyses stratégiques incomplètes et parfois biaisées.

Si la volonté de simplifier un modèle ne dénature pas les principes de l'ABC et permet d'orienter la décision stratégique, les limites révélées témoignent de l'équilibre difficile à trouver entre souplesse d'utilisation et recherche d'informations fines et précises. Dans le premier cas le niveau de technicité du modèle est délibérément restreint, les concepteurs ayant exclu de systématiser les procédures pour valider les niveaux d'homogénéité des ensembles de coûts (maille d'analyse) ou pour tester les causalités entre inducteurs. Les dispositifs décrits ont pour objectif de redéfinir certaines des relations avec les clients en modifiant notamment les offres tarifaires, ce qui témoigne du potentiel stratégique du modèle. $\mathrm{Ce}$ potentiel stratégique est aussi avéré dans le second cas puisque le déploiement de l'ABC a permis de calculer des coûts unitaires par type de crédits et par type de clients, ce qui permet d'aider à la prise de décision en matière de politique tarifaire (validation des hypothèses stratégiques : pilotage stratégique des coûts version médiane). Pour les deux groupes, l'ABC a été l'occasion d'élaborer des benchmarks. Il s'agit d'utiliser l'ABC pour comparer les coûts de différentes solutions informatiques, de façon à proposer aux clients le meilleur service possible à un coût maîtrisé.

Ces deux études de cas montrent au final qu'il est possible d'élaborer des systèmes de pilotage stratégique à base d'activités pour des services informatiques, en gardant à l'esprit les limites que nous venons de souligner.

\section{Conclusion}

Cet article a été l'occasion de faire le point sur le pilotage stratégique des coûts en nous centrant les approches à base d'activités. Nous nous sommes demandé si les développements et les études de cas exposés dans cet article valident le potentiel stratégique de ces approches.

En nous fondant sur une grille de lecture des motifs d'usage de l'ABC, nous avons montré que la dimension stratégique est au cour de plusieurs développements sur l'ABC. Sur la base des travaux décrits, on peut donc dire que l'ABC fait partie de la famille des outils de pilotage stratégique des coûts. Tout en soulignant les limites de l'outil, nous avons constaté qu'il a pour objectif d'aider les managers à valider leurs hypothèses stratégiques. Pour la partie consacrée aux études de cas, nous constatons au final que les deux modèles déployés ont une orientation stratégique marquée et que l'ABC mis en place au sein du groupe 2 est plus complexe. Cette complexité, notamment dans la multiplication des métriques utilisées pour répartir les prestations et les activités informatiques, permet de rassurer les clients, rendant moins discutables les propositions tarifaires. Observons en revanche que le nombre limité d'inducteurs dans le modèle du groupe 1 a généré des ruptures dans l'explication des liens de causalité entre la génération des coûts et leurs déterminants.

Notre recherche mériterait d'être complétée par d'autres études de cas mettant en scène des déploiements d'ABC dans d'autres secteurs. Enfin, un autre prolongement serait de davantage centrer nos travaux sur les questions soulevées par les dynamiques organisationnelles en focalisant nos observations sur les acteurs qui participent au processus et sur ceux qui le subissent. 


\section{Bibliographie}

Anderson S.W. et Young S.M. (1999), «The Impact of Contextual and Process Factors on the Evaluation of Activity-Based Costing Systems », Accounting Organizations and Society, Vol. $24, \mathrm{~N}^{\circ} 7$, p. 525-559.

Anthony R.N. (1965), Planning and Control Systems: A Framework for Analysis, Division of Research, Graduate School of Business Administration, Harvard University, Boston, Mass.

Antos J. et Brimson J.A. (1999), Driving value using activity-based budgeting, John Wiley \& sons, New York.

Argyris Ch. et Schön D.A. (1978), Organizational Learning, Readings, Addison-Wesley Publishing Company.

Berland N. et Gervais M. (2008), «A quoi ont rêvé (et n'ont pas rêvé) les chercheurs en contrôle durant les dix dernières années ? Dix ans de recherche en contrôle », Finance Contrôle Stratégie, Vol. 11 (hors série), p. 109 - 148.

Bouquin H. (2006), Comptabilité de Gestion, $4^{\text {ème }}$ édition, Economica, Paris.

Brickley J.A. et al. (1997), Managerial Economics and Organizational Architecture, McGraw Hill.

Bromwich M. (1990), « The Case for Strategic Management Accounting: The Role of Accounting Information for Strategy in Competitive Markets », Accounting Organizations and Society, Vol. 15 (1/2), p. 127-146.

Caglio A. et Ditillo A. (2008), «A Review and Discussion of Management Control in Interfirm Relationships: Achievements and Future Directions », Accounting Organizations and Society, Vol. 33, p. 865-898.

Chatelain-Ponroy S. et Sponem S. (2007), «Evolutions et permanence du contrôle de gestion », Economie et Management, $\mathrm{N}^{\circ} 123$, avril, p. 12-18.

Cokins G. (2002), «Integrating Target Costing and ABC », Journal of Cost Management, July-August, p. 13-22.

Cooper R.et Kaplan R.S. (1999), The Design of Cost Management Systems, Prentice Hall.

Cooper R.et Kaplan R.S. (1988), « Measure Costs Right: Make the Right Decisions », Harvard Business Review, September-October, p. 96-103.

Cooper R. et Slagmulder R. (2004), «Interorganizational Cost Management and Relational Context », Accounting Organization and Society, Vol. 29, p. 1-26.

Datar S. et Gupta M. (1994), « Aggregation, Specification and Measurement Errors in Product Costing », The Accounting Review, Vol. 69, N 4, p. 567-591.

Foster G. et Swenson D.W. (1997), « Measuring the Success of Activity-Based Cost Management and Its Determinants », Journal of Management Accounting Research, Vol. 9, p. 109-141.

Gervais M., Levant Y., Ducrocq C. (2010), «Le Time-Driven Activity-Based Costing (TDABC) : un premier bilan à travers une étude de cas longitudinale », Finance Contrôle Stratégie, vol. 13, $\mathrm{n}^{\circ}$ 1, mars 2010, p. 123-155.

Gervais M. et Lesage C. (2006), «Retour sur l'imputation des charges indirectes en comptabilité de gestion: comment bien spécifier les activités et leurs inducteurs?», Comptabilité Contrôle Audit, Vol. 12, № 1, p. 85-101.

Hamel G. et Prahalad C.K. (1990), «The Core Competence of the Corporation », Harvard Business Review, Vol. 68, N 3, p. 79-92.

Hoffjan A. et Wömpener A. (2006), « Comparative Analysis of Strategic Management Accounting in German - and English - Language General Management Accounting Textbooks », Schmalenbach Business Review, Vol. 58, p. 234-258.

Horngren C.T. et al. (2005), Cost Accounting - A Managerial Emphasis, $12^{\text {th }}$ edition, Pearson Education.

Horvath P. et al. (1998), « Linking Target Costing to ABC at a US Automative Supplier », 
Journal of Cost Management, Vol. 12, $\mathrm{N}^{\circ} 2$, p. 16-24.

Jensen M.C. et Meckling W.H. (1992), « Specific and General Knowledge, and Organizational Structure », dans L., Werin; H., Wijkander (sous la direction de), Contract Economics, Blackwell.

Johnson H.T. et Bröms A. (2002), La méthode MBM; pour un management de la performance durable, Editions de l'Organisation, Paris.

Johnson H.T. et Kaplan R.S. (1987), Relevance Lost: The Rise and Fall of Management Accounting Systems, Harvard Business School Press, Boston.

Jones T.C. et Dugdale D. (2002), «The ABC Bandwagon and the Juggernaut of Modernity », Accounting Organizations and Society, Vol. 27, p. 121-163.

Kaplan R.S. et Anderson S.R. (2007), Time-driven Activity-based Costing, Harvard Business School Press, Boston.

Keys D.E. et van der Merwe A. (2002), "The Case for Resource Consumption Accounting », Strategic Finance, Vol. 83, № 10 et 11, p. 30-36 et 41-47.

Kuchta D. et Troska M. (2007), « Activity-based Costing and Customer Profitability », Cost Management, Vol. 21, $\mathrm{N}^{\circ} 3$, p. 18-25.

La villarmois O. (de) et Levant Y. (2007), «Le Time-Driven ABC: la simplification de l'évaluation des coûts par le recours aux équivalents - un essai de positionnement », Finance Contrôle Stratégie, Vol. 10, N 1, p. 149-182.

Lebas M. (1999), «Why ABC? Accounting Based on Causality Rather than Activity-Based Costing », European Management Journal, Vol. 17, N 5, p. 501-511.

Le Moigne J.L. (1996), « Les deux sources de la performance des organisations : cohérence du contrôle, impertinence de l'intelligence » in Jacot J.H. et Lorino Ph (dir.), Cohérence, Pertinence et Evaluation, Ecosip, Economica, Paris, p 31-45.

Lorino P. (1991), Le pilotage stratégique des coûts, Dunod, Paris.

Malmi T. (1997), « Towards explaining Activity-Based Costing Failure: Accounting and Control in a Decentralised Organization », Management Accounting Research, Vol. 8, p. 459480.

Mévellec P. (2005), Les systèmes de coûts. Objectifs, paramètres de conception et analyse comparée, Dunod, Paris.

Sabbah S. (2009), «Optimiser les coûts informatiques : un exercice difficile mais possible », Revue Echanges, février, p. 46-48.

Savall H. et Zardet V. (2010), Maîtriser les coûts et les performances cachées, $5^{\mathrm{e}}$ édition, Economica, Paris.

Shank J. et Govindarajan V. (1989), Strategic cost analysis: the evolution from managerial to strategic accounting, Burn Ridge Inc, Illinois.

Simmonds K. (1981), «Strategic Management Accounting », Management Accounting, Vol 59, $\mathrm{N}^{\circ} 4$, p. 26-29.

Simons R.L. (1995), Levers of Control, Harvard Business School Press, Boston.

Teller R. (1999), Le contrôle de gestion, pour un pilotage intégrant stratégie et finance, Management et Société, Paris.

Thomas C. et Gervais M. (2008), «Le problème du regroupement des activités dans la modélisation ABC. Une approche possible », Finance Contrôle Stratégie, Vol. 11, N 4, p. 137-170.

Tomkins C. et Carr C. (sous la direction de) (1996), « Special Issue on Strategic Management Accounting », Management Accounting Research, Vol. 7, p. 165-167. 